Interfaces and Free Boundaries 15 (2013), 77-93

DOI 10.4171/IFB/295

\title{
Polygonal Hele-Shaw problem with surface tension
}

\author{
MASATO KIMURA \\ Faculty of Mathematics and Physics, Institute of Science and Engineering, Kanazawa University \\ Kakuma, Kanazawa, 920-1192, Japan \\ E-mail:mkimura@se.kanazawa-u.ac.jp \\ DAISUKE TAGAMI \\ Institute of Mathematics for Industry, Kyushu University 744 Motooka, Nishi-ku, Fukuoka, \\ 819-0395, Japan \\ E-mail:tagami@imi.kyushu-u.ac.jp \\ SHIGETOSHI YAZAKI \\ Department of Mathematics, School of Science and Technology, Meiji University 1-1-1 \\ Higashi-Mita, Tama-ku, Kawasaki-shi, Kanagawa 214-8571, Japan \\ E-mail:syazaki@meiji.ac.jp
}

[Received 29 September 2011 and in revised form 7 September 2012]

\begin{abstract}
We study a polygonal analogue of the Hele-Shaw moving boundary problem with surface tension based on a framework of polygonal motion proposed by Beneš et al. [5]. A key idea is to introduce a polygonal Dirichlet-to-Neumann map. We study variational properties of the polygonal Dirichletto-Neumann map and show that our polygonal Hele-Shaw problem is a polygonal analogue of the original problem. Local solvability of a polygonal Hele-Shaw problem is also proved by means of the variational structure.
\end{abstract}

2010 Mathematics Subject Classification: Primary 35R35, 76D27; Secondary 53C44.

Keywords: Motion of polygons; moving boundary problem; polygonal Hele-Shaw problem; variational structure; Dirichlet-to-Neumann map, local solvability.

\section{Introduction}

We consider a mathematical model for moving boundaries in one phase Hele-Shaw flow. The HeleShaw flow is two-dimensional slow flow of viscous fluid between two parallel horizontal plates with a thin gap. It was studied by H. S. Hele-Shaw [14] experimentally at first, and some mathematical models have been proposed in several papers [20-22, 24].

We suppose that the viscous fluid occupies a bounded domain $\Omega(t) \subset \mathbb{R}^{m}$ at time $t$. Although the Hele-Shaw flow is a two dimensional flow, we consider our mathematical model in general dimension $m \geqslant 2$. We assume that $\Gamma(t):=\partial \Omega(t)$ is sufficiently smooth and moves smoothly. We suppose that the outward normal velocity of $\Gamma(t)$ is denoted by $V(\boldsymbol{x}, t)$. A standard mathematical 
model is as follows:

$$
\begin{cases}\boldsymbol{u}(\boldsymbol{x}, t)=-\nabla p(\boldsymbol{x}, t) & (\boldsymbol{x} \in \Omega(t), t \in[0, T)) \\ \operatorname{div} \boldsymbol{u}(\boldsymbol{x}, t)=0 & (\boldsymbol{x} \in \Omega(t), t \in[0, T)) \\ p(\boldsymbol{x}, t)=\sigma \kappa(\boldsymbol{x}, t) & (\boldsymbol{x} \in \Gamma(t), t \in[0, T)) \\ V(\boldsymbol{x}, t)=\boldsymbol{n} \cdot \boldsymbol{u}(\boldsymbol{x}, t) & (\boldsymbol{x} \in \Gamma(t), t \in[0, T)) \\ \Gamma(0)=\Gamma_{0}, & \end{cases}
$$

where $p(\boldsymbol{x}, t) \in \mathbb{R}$ and $\boldsymbol{u}(\boldsymbol{x}, t) \in \mathbb{R}^{m}(\boldsymbol{x} \in \Omega(t))$ are a normalized pressure field and a velocity field, respectively. The velocity field is proportional to the gradient of pressure [20]. The third equation represents a surface tension effect, where $\sigma>0$ is a surface tension which is a given fixed constant and $\kappa$ denotes sum of principal curvatures of $\Gamma(t)$. If $\Omega(t)$ is convex then $\kappa \geqslant 0$.

The Hele-Shaw problem is one of the simplest typical moving boundary problems and it admits several variational structures which will be shown in Theorem 2.4 below. So far, there are many different mathematical approaches to analyze this problem. Duchon and Robert [7] gave a mathematical interpretation of surface tension as corresponding to the coefficient of the principal part of a nonlinear third order parabolic integro-differential equation and proved a time local existence of the unique solution in graph setting without source term. The parabolicity of the equation is a mathematical expression of the stabilizing effect of surface tension. Their result was then extended in [16] to more wider class of geometry with source terms. A mathematical relation between the fingering phenomena and the surface tension effect was also discussed in terms of mathematical ill/well-posedness in [16]. Escher and Simonett [8] studied the multi-dimensional case using an abstract theory for nonlinear parabolic semigroups and proved short-time existence of the moving boundary with a driving force. Chen et al. [6] also proved existence of the solution of the Hele-Shaw problem with surface tension by a forth order regularization technique.

The variational structure of the Hele-Shaw problem often plays an essential role not only in the analysis but also in the numerical analysis. For instance, preservation of such variational structures in numerical simulation deeply concerns its stability and accuracy. Although there are a great number of numerical schemes for this problem (see [1, 15, 23] and references therein), most of them realize variational structures approximately. In contrast to them, our polygonal analogue Hele-Shaw flow preserves the variational structures and is regarded as a semi-discrete numerical scheme, that is, it is continuous in time and discrete in space.

Beneš et al. [5] proposed a polygonal motion in $\mathbb{R}^{2}$ with a notion of a polygonal curvature. It is a generalization of the crystalline motion which is a mathematical model of crystal growth. They applied their polygonal motion not only to the curvature flow but also to wider classes of moving boundary problems and showed that the polygonal motion preserves the original variational structures almost perfectly.

In this paper, we consider a polygonal Hele-Shaw problem based on the idea of [5], and show that it is a polygonal analogue of the original problem (1.1). This is not the first attempt to apply the crystalline motion to the Hele-Shaw problem (see [3, 10, 11]). Particularly in [10, 11], they proposed a crystal growth model of a three dimensional cylindrical domain similar to our polygonal Hele-Shaw problem, and they made good use of the variational structures. Our motivation, however, is different from these papers, because our view point is to construct polygonal version of a moving boundary problem preserving the variational structures, and we have chosen the Hele-Shaw problem as typical model.

The outline of this paper is as follows. In Section 2, we review a standard Dirichlet-to-Neumann map in a smooth domain and show the variational structures of the Hele-Shaw problem such as 
volume preservation and surface decreasing properties. It is also shown that the center of gravity does not move. In Section 3, we consider polygonal motions mainly in two dimensional space. We study some properties of a polygonal Dirichlet-to-Neumann map in Section 3.1, and we briefly review the polygonal motion proposed in [5] in Section 3.2. In Section 3.3, we construct a polygonal version of the Hele-Shaw problem and show its variational structure. Finally, time local solvability and uniqueness of the solution of polygonal Hele-Shaw problem are proved in Section 4.

\section{Motion of smooth surfaces}

\subsection{Dirichlet-to-Neumann map}

As a preliminary in studying the Hele-Shaw problem, we define the Dirichlet-to-Neumann map and study its properties. Let $\Omega$ be a bounded Lipschitz domain in $\mathbb{R}^{m}(m \in \mathbb{N}, m \geqslant 2)$ with its boundary $\Gamma:=\partial \Omega$. We define a bilinear form on the Sobolev space $H^{1}(\Omega)$ by

$$
a(u, v):=\int_{\Omega} \nabla u \cdot \nabla v d \boldsymbol{x} \quad\left(u, v \in H^{1}(\Omega)\right) .
$$

For a function $v \in H^{1}(\Omega)$, we define a trace operator $\gamma_{0} v:=\left.v\right|_{\Gamma}$. It is known that $\gamma_{0}$ is a bounded operator from $H^{1}(\Omega)$ onto $H^{\frac{1}{2}}(\Gamma)$ (see [13]). We denote the dual space of $H^{\frac{1}{2}}(\Gamma)$ by $H^{-\frac{1}{2}}(\Gamma):=$ $\left(H^{\frac{1}{2}}(\Gamma)\right)^{\prime}$. For $g \in H^{\frac{1}{2}}(\Gamma)$, there exists a unique weak solution $u \in H^{1}(\Omega)$ to the following boundary value problem:

$$
\begin{cases}\Delta u=0 & \text { in } \Omega \\ u=g & \text { on } \Gamma\end{cases}
$$

We define an affine space:

$$
X(g):=\left\{v \in H^{1}(\Omega) ; \gamma_{0} v=g\right\} \quad\left(g \in H^{\frac{1}{2}}(\Gamma)\right) .
$$

A weak form of (2.1) is as follows:

$$
u \in X(g) \quad \text { s.t. } \quad a(u, v)=0 \quad\left({ }^{\forall} v \in X(0)\right),
$$

and it is equivalent with the following minimization problem:

$$
\text { Find } u=\underset{v \in X(g)}{\arg \min } a(v, v) \text {, }
$$

which means

$$
\text { Find } u \in X(g) \quad \text { s.t. } \quad a(u, u) \leqslant a(v, v) \quad\left({ }^{\forall} v \in X(g)\right) \text {. }
$$

It is well-known that there exists a unique solution to (2.2) or (2.3). We consider a Dirichlet-toNeumann map $\Lambda_{\Gamma}$ by

$$
\Lambda_{\Gamma} g=\frac{\partial u}{\partial \boldsymbol{n}}
$$


where $u$ is the weak solution of (2.2) and $\boldsymbol{n}$ denotes the outward unit normal vector on $\Gamma$. Formally, for an arbitrary $v \in H^{1}(\Omega)$, it satisfies

$$
\int_{\Gamma}\left(\Lambda_{\Gamma} g\right) v d H^{m-1}=\int_{\Gamma} \frac{\partial u}{\partial \boldsymbol{n}} v d H^{m-1}=\int_{\Omega} \operatorname{div}(v \nabla u) d \boldsymbol{x}=a(u, v),
$$

where $d H^{m-1}$ denotes the $(m-1)$-dimensional Hausdorff measure. A precise definition and properties of $\Lambda_{\Gamma}$ are given in the next theorem. We omit its proof, but it is not difficult to show the following theorem from a standard argument as in [13].

THEOREM 2.1 (Dirichlet-to-Neumann map) There exists $\Lambda_{\Gamma} \in B\left(H^{\frac{1}{2}}(\Gamma), H^{-\frac{1}{2}}(\Gamma)\right)$ such that

$$
H^{-\frac{1}{2}(\Gamma)}\left\langle\Lambda_{\Gamma} g, \gamma_{0} v\right\rangle_{H^{\frac{1}{2}}(\Gamma)}=a(u, v) \quad\left({ }^{\forall} g \in H^{\frac{1}{2}}(\Gamma),{ }^{\forall} v \in H^{1}(\Omega)\right),
$$

where $u$ is the solution of (2.2). Moreover, $\Lambda_{\Gamma}$ is a symmetric operator, i.e., $\Lambda_{\Gamma}^{\prime}=\Lambda_{\Gamma}$ and satisfies

$$
\begin{aligned}
& \operatorname{Ker}\left(\Lambda_{\Gamma}\right)=\left\{g \in H^{\frac{1}{2}}(\Gamma) ; g \equiv \text { const. }\right\} \\
& \operatorname{Im}\left(\Lambda_{\Gamma}\right)=\left\{\lambda \in H^{-\frac{1}{2}}(\Gamma) ; H^{-\frac{1}{2}(\Gamma)}\langle\lambda, 1\rangle_{H^{\frac{1}{2}}(\Gamma)}=0\right\} .
\end{aligned}
$$

\subsection{General motion and variational formulas}

We consider motion of smooth hypersurfaces in $\mathbb{R}^{m}(m \in \mathbb{N}, m \geqslant 2)$ in this section. Let $\Omega(t)$ be a bounded domain in $\mathbb{R}^{m}$ moving in time $t \in I$, where $I$ is an interval. We denote the boundary of $\Omega(t)$ by $\Gamma(t)$ and call $M:=\bigcup_{t \in I}(\Gamma(t) \times\{t\})$ a moving boundary. We suppose that $M$ is sufficiently smooth, e.g., $M$ is a $C^{2,1}$-class moving boundary (which means the outward unit normal vector field $\boldsymbol{n}$ of $\Gamma(t)$ belongs to $C^{1}\left(M ; \mathbb{R}^{m}\right)$ [18]). Then we can define the outward normal velocity $V \in C^{0}(M ; \mathbb{R})$ and sum of principal curvatures $\kappa \in C^{0}(M ; \mathbb{R})$.

The $m$-dimensional measure of $\Omega(t)$ and the $(m-1)$-dimensional Hausdorff measure of $\Gamma(t)$ are denoted by $|\Omega(t)|$ and $|\Gamma(t)|$, respectively. We define the center of gravity of $\Omega(t)$ by

$$
c(t):=\frac{1}{|\Omega(t)|} \int_{\Omega(t)} \boldsymbol{x} d \boldsymbol{x} .
$$

The following results are easily derived from standard variational formulas. See [18] for further details.

THEOREM 2.2 If $M$ is a $C^{2,1}$-class moving boundary, then $|\Omega(t)|,|\Gamma(t)|$ and $c(t)$ belong to $C^{1}(I)$ with respect to $t$ and the following formulas hold for $t \in I$.
1. $\frac{d}{d t}|\Omega(t)|=\int_{\Gamma(t)} V d H^{m-1}$.
2. $\frac{d}{d t}|\Gamma(t)|=\int_{\Gamma(t)} \kappa V d H^{m-1}$.

3. If $|\Omega(t)|$ is constant in time, $\frac{d}{d t} c(t)=\frac{1}{|\Omega|} \int_{\Gamma(t)} x V d H^{m-1}$. 


\subsection{Hele-Shaw problem with surface tension}

In this section, we consider a $C^{2,1}$-class moving boundary

$$
M:=\bigcup_{0 \leqslant t<T}(\Gamma(t) \times\{t\})
$$

and suppose that, for each $t \in[0, T), \Gamma(t)$ is a boundary of a bounded domain $\Omega(t) \subset \mathbb{R}^{m}$. For simplicity, we write $V(t):=\left.V\right|_{\Gamma(t)}$ and $\kappa(t):=\left.\kappa\right|_{\Gamma(t)}$. We denote the bilinear forms on $\Omega(t)$ and $\Gamma(t)$ by

$$
a_{t}(u, v):=\int_{\Omega(t)} \nabla u \cdot \nabla v d \boldsymbol{x}, \quad\langle u, v\rangle_{t}:=_{H^{-\frac{1}{2}}(\Gamma(t))}\langle u, v\rangle_{H^{\frac{1}{2}}(\Gamma(t))} .
$$

The trace operator from $H^{1}(\Omega(t))$ to $H^{\frac{1}{2}}(\Gamma(t))$ is, however, always denoted just by $\gamma_{0}$.

The Hele-Shaw problem (1.1) is described by using the Dirichlet-to-Neumann map.

Problem 2.3 For a given bounded domain $\Omega_{0}$ in $\mathbb{R}^{m}$ with a smooth boundary $\Gamma_{0}=\partial \Omega_{0}$, find a $C^{2,1}$-class moving boundary $M$ with $\kappa(t) \in H^{\frac{1}{2}}(\Gamma(t))$ for $t \in[0, T)$ such that

$$
\left\{\begin{array}{l}
V(t)=-\sigma \Lambda_{\Gamma(t)}(\kappa(t)) \quad(t \in[0, T)) \\
\Gamma(0)=\Gamma_{0}
\end{array}\right.
$$

Under the condition (2.4), for each $t \in[0, T)$, the pressure field $p(t)=p(\cdot, t)$ and the velocity field $\boldsymbol{u}(t)=\boldsymbol{u}(\cdot, t)$ are constructed in the following spaces.

$$
\begin{aligned}
& p(t) \in H^{1}(\Omega(t)) \quad \text { s.t. } \quad \begin{cases}a_{t}(p(t), v)=0 & \left(v \in H_{0}^{1}(\Omega(t))\right) \\
\gamma_{0} p(t)=\sigma \kappa(t) & \text { on } \Gamma(t)\end{cases} \\
& \boldsymbol{u}(t)=-\nabla p(t) \in L^{2}\left(\Omega(t) ; \mathbb{R}^{m}\right)
\end{aligned}
$$

The solution satisfies the following properties.

THEOREM 2.4 If $M$ is a solution of Problem 2.3, the following properties hold.

1. $\frac{d}{d t}|\Omega(t)|=0$

2. $\frac{d}{d t}|\Gamma(t)|=-\frac{1}{\sigma} \int_{\Omega(t)}|\boldsymbol{u}(\boldsymbol{x}, t)|^{2} d \boldsymbol{x} \leqslant 0$

3. $\frac{d}{d t} c(t)=\mathbf{0}$

Proof. From the formulas of Theorem 2.2, we obtain the area preserving property:

$$
\frac{d}{d t}|\Omega(t)|=\int_{\Gamma(t)} V d H^{m-1}=\langle V(t), 1\rangle_{t}=\left\langle-\sigma \Lambda_{\Gamma(t)}(\kappa(t)), 1\right\rangle_{t}=-a_{t}(p(t), 1)=0 .
$$

The second property also follows.

$$
\begin{aligned}
\frac{d}{d t}|\Gamma(t)| & =\int_{\Gamma(t)} \kappa V d H^{m-1}=-\left\langle\Lambda_{\Gamma(t)}(\kappa(t)), \gamma_{0} p(t)\right\rangle_{t} \\
& =-\frac{1}{\sigma} a_{t}(p(t), p(t))=-\frac{1}{\sigma} \int_{\Omega(t)}|\boldsymbol{u}(\boldsymbol{x}, t)|^{2} d \boldsymbol{x}
\end{aligned}
$$


For the center of gravity, from property 1, we can use formula 3 of Theorem 2.2. Let $c(t)=$ $\left(c_{1}(t), \cdots, c_{m}(t)\right)^{\mathrm{T}}$. We define an identity map $\boldsymbol{\beta}(\boldsymbol{x}):=\boldsymbol{x}$, and $\beta_{i}(\boldsymbol{x}):=x_{i}$. For $i=1,2, \cdots, m$, we have

$$
\begin{aligned}
|\Omega(0)| \frac{d}{d t} c_{i}(t) & =\int_{\Gamma(t)} \beta_{i} V d H^{m-1}=-\left\langle\sigma \Lambda_{\Gamma(t)}(\kappa(t)), \beta_{i}\right\rangle_{t} \\
& =-a_{t}\left(p(t), \beta_{i}\right)=-\int_{\Omega(t)} \frac{\partial p}{\partial x_{i}}(\boldsymbol{x}, t) d \boldsymbol{x} .
\end{aligned}
$$

Hence, we have

$$
|\Omega(0)| \frac{d}{d t} c(t)=-\int_{\Omega(t)} \nabla p(\boldsymbol{x}, t) d \boldsymbol{x}=-\int_{\Gamma(t)} p \boldsymbol{n} d H^{m-1}=-\sigma \int_{\Gamma(t)} \kappa \boldsymbol{n} d H^{m-1}=0 .
$$

The last equality holds for any bounded $C^{2}$ closed hypersurface $\Gamma$. We have used the formula:

$$
\int_{\Gamma}\left(\Delta_{\Gamma} u\right) v d H^{m-1}=-\int_{\Gamma} \nabla_{\Gamma} u \cdot \nabla_{\Gamma} v d H^{m-1} \quad\left({ }^{\forall} u \in C^{2}(\Gamma),{ }^{\forall} v \in C^{1}(\Gamma)\right),
$$

where $\Delta_{\Gamma}$ and $\nabla_{\Gamma}$ are the Laplace-Beltrami operator and the gradient operator on $\Gamma$, respectively (see [18]). In particular, if $v \equiv 1$, we have

$$
\int_{\Gamma} \Delta_{\Gamma} u d H^{m-1}=0 \quad\left({ }^{\forall} u \in C^{2}(\Gamma)\right) .
$$

Since $\Delta_{\Gamma} \boldsymbol{\beta}=-\kappa \boldsymbol{n}$ holds on $\Gamma$ [18], we have

$$
\int_{\Gamma} \kappa \boldsymbol{n} d H^{m-1}=-\int_{\Gamma} \Delta_{\Gamma} \boldsymbol{\beta} d H^{m-1}=0
$$

\section{Motion of polygons}

In the following two sections, we proceed the argument in two dimensional case.

\subsection{Polygonal Dirichlet-to-Neumann map}

For a polygonal domain, we define a discrete Dirichlet-to Neumann map for our construction of a polygonal Hele-Shaw problem. Let $\Omega$ be a bounded polygonal domain in $\mathbb{R}^{2}$ with a polygonal boundary $\Gamma=\partial \Omega$. We suppose $\Gamma$ is an $N$-polygon and the $N$ edges (open line segments) are denoted by $\Gamma_{j}$ for $(j=1,2, \cdots, N)$. We denote the characteristic function of $\Gamma_{j}$ by

$$
\chi_{j}(x)= \begin{cases}1 & \left(x \in \Gamma_{j}\right), \\ 0 & \left(x \in \Gamma \backslash \Gamma_{j}\right),\end{cases}
$$

and define an $N$-dimensional subspace of $L^{2}(\Gamma)$ as

$$
W(\Gamma):=\operatorname{span}\left\langle\chi_{1}, \chi_{2}, \cdots, \chi_{N}\right\rangle \subset L^{2}(\Gamma) .
$$


We define a polygonal trace operator $\bar{\gamma}_{0}: H^{1}(\Omega) \rightarrow W(\Gamma)$ by

$$
\bar{\gamma}_{0} v:=\sum_{j=1}^{N}\left(f_{\Gamma_{j}} v d H^{1}\right) \chi_{j} \in W(\Gamma) \quad\left(v \in H^{1}(\Omega)\right),
$$

where $f_{\Gamma_{j}} \mathrm{~F} d H^{1}:=\left(\int_{\Gamma_{j}} \mathrm{~F} d H^{1}\right) /\left|\Gamma_{j}\right|$ and $\left|\Gamma_{j}\right|:=H^{1}\left(\Gamma_{j}\right)$.

We recall that the purpose of this paper is to analyze a polygonal version of the original HeleShaw problem (1.1), which will be formulated as Problem 3.6 below. To this end, we propose a polygonal version of (2.1) as follows. For given $\bar{g} \in W(\Gamma)$, we consider the following boundary value problem in $\Omega$ :

$$
\begin{cases}\Delta u=0 & \text { in } \Omega \\ \bar{\gamma}_{0} u=\bar{g} & \text { on } \Gamma \\ \left.\frac{\partial u}{\partial \boldsymbol{n}_{j}}\right|_{\Gamma_{j}}=\text { const. } & (j=1,2, \cdots, N),\end{cases}
$$

where $\boldsymbol{n}_{j} \in \mathbb{R}^{2}$ denotes the outward unit normal vector of $\Gamma_{j}$.

For $\bar{g} \in W(\Gamma)$, we define an affine subspace of $H^{1}(\Omega)$ :

$$
X_{N}(\bar{g}):=\left\{v \in H^{1}(\Omega) ; \bar{\gamma}_{0} v=\bar{g}\right\},
$$

and define $X_{N}:=X_{N}(0)$. A weak formulation of the problem (3.1) is given as follows:

$$
\text { Find } u \in X_{N}(\bar{g}) \quad \text { s.t. } \quad\left\{\begin{array}{l}
a(u, v)=0 \\
\Lambda_{\Gamma}\left(\gamma_{0} u\right) \in W(\Gamma) .
\end{array}\left(^{\forall} v \in H_{0}^{1}(\Omega)\right),\right.
$$

We can construct an equivalent minimization problem similarly to (2.2):

$$
\text { Find } \quad u=\underset{v \in X_{N}(\bar{g})}{\arg \min } a(v, v)
$$

Theorem 3.1 Problem (3.2) has a solution $u \in X_{N}(\bar{g})$ and it is unique. Moreover, the solution $u$ is a unique minimizer of (3.3).

Proof. We denote the dual map $H^{-\frac{1}{2}(\Gamma)}\langle\cdot, \cdot\rangle_{H^{\frac{1}{2}}(\Gamma)}$ simply by $\langle\cdot, \cdot\rangle$. We first prove the uniqueness of a solution of (3.2). Let $u_{1} \in X_{N}(\bar{g})$ and $u_{2} \in X_{N}(\bar{g})$ be solutions of (3.2) and let $w:=u_{1}-u_{2}$. We remark that $w \in X_{N}, a(w, v)=0$ for $v \in H_{0}^{1}(\Omega)$, and $\Lambda_{\Gamma}\left(\gamma_{0} w\right)=\sum_{j=1}^{N} \mu_{j} \chi_{j}$, where $\mu_{j} \in \mathbb{R}$. From these relations, we obtain

$$
a(w, w)=\left\langle\Lambda_{\Gamma}\left(\gamma_{0} w\right), \gamma_{0} w\right\rangle=\sum_{j=1}^{N} \mu_{j}\left\langle\chi_{j}, \gamma_{0} w\right\rangle=\sum_{j=1}^{N} \mu_{j} \int_{\Gamma_{j}} w d H^{1}=0,
$$

and it implies that $w=0$, namely, $u_{1}=u_{2}$.

Since $X_{N}(\bar{g})$ is a closed affine subspace in $H^{1}(\Omega)$, it is clear that there exists a unique minimizer $u \in X_{N}(\bar{g})$ of problem (3.3). Its first variation gives the following weak form:

$$
a(u, v)=0 \quad\left({ }^{\forall} v \in X_{N}\right) .
$$


We define its Neumann boundary value $\lambda:=\Lambda_{\Gamma}\left(\gamma_{0} u\right) \in H^{-\frac{1}{2}}(\Gamma)$. From the definition of the Dirichlet-to-Neumann map,

$$
\left\langle\lambda, \gamma_{0} v\right\rangle=a(u, v) \quad\left({ }^{\forall} v \in H^{1}(\Omega)\right)
$$

holds. For each $j=1,2, \cdots, N$, we choose $v_{j} \in X_{N}\left(\chi_{j}\right)$ and define

$$
\lambda_{j}:=\frac{1}{\left|\Gamma_{j}\right|}\left\langle\lambda, \gamma_{0} v_{j}\right\rangle \quad(j=1,2, \cdots, N) .
$$

For arbitrary $v \in H^{1}(\Omega)$, we define $p_{j}:=f_{\Gamma_{j}} v d H^{1}$ and $\tilde{v}:=\sum_{j=1}^{N} p_{j} v_{j}$. Since $v-\tilde{v} \in X_{N}$, from (3.4), we have

$$
\begin{aligned}
\left\langle\lambda, \gamma_{0} v\right\rangle & =a(u, v)=a(u, \tilde{v})=\left\langle\lambda, \gamma_{0} \tilde{v}\right\rangle=\sum_{j=1}^{N} p_{j}\left\langle\lambda, \gamma_{0} v_{j}\right\rangle \\
& =\sum_{j=1}^{N} \lambda_{j} p_{j}\left|\Gamma_{j}\right|=\sum_{j=1}^{N} \lambda_{j} \int_{\Gamma_{j}} v d H^{1}=\left\langle\sum_{j=1}^{N} \lambda_{j} \chi_{j}, \gamma_{0} v\right\rangle .
\end{aligned}
$$

Hence,

$$
\Lambda_{\Gamma}\left(\gamma_{0} u\right)=\sum_{j=1}^{N} \lambda_{j} \chi_{j} \in W(\Gamma),
$$

holds and $u$ is a unique solution of problem (3.2). This also shows that (3.3) admits a unique minimizer.

DEFINITION 3.2 (Polygonal Dirichlet-to-Neumann map) Let $\Gamma$ be a bounded $N$-polygon in $\mathbb{R}^{2}$. We define a polygonal Dirichlet-to-Neumann map $\bar{\Lambda}_{\Gamma}$, which is a linear operator from $W(\Gamma)$ to $W(\Gamma)$, as follows. For $\bar{g} \in W(\Gamma)$ and for a solution $u \in X_{N}(\bar{g})$ of (3.2), we define $\bar{\Lambda}_{\Gamma}$ and its $j$-th component $\Lambda_{j}$ by

$$
\bar{\Lambda}_{\Gamma}(\bar{g}):=\sum_{j=1}^{N} \Lambda_{j}(\bar{g}) \chi_{j}:=\Lambda_{\Gamma}\left(\gamma_{0} u\right) \in W(\Gamma) .
$$

The following theorem is a polygonal analogue of Theorem 2.1.

TheOREM 3.3 The polygonal Dirichlet-to-Neumann map $\bar{\Lambda}_{\Gamma}$ is symmetric with respect to $L^{2}(\Gamma)$ inner product, i.e.,

$$
\left(\bar{\Lambda}_{\Gamma}(\bar{g}), \bar{h}\right)_{L^{2}(\Gamma)}=\left(\bar{g}, \bar{\Lambda}_{\Gamma}(\bar{h})\right)_{L^{2}(\Gamma)} \quad(\bar{g}, \bar{h} \in W(\Gamma)) .
$$

Furthermore, it has the following properties:

$$
\Lambda_{j}(\bar{g})=\frac{a\left(u, v_{j}\right)}{\left|\Gamma_{j}\right|} \quad\left(\bar{g} \in W(\Gamma), v_{j} \in X_{N}\left(\chi_{j}\right), j=1,2, \cdots, N\right),
$$

where $u$ is a solution of (3.2), and

$$
\operatorname{Ker}\left(\bar{\Lambda}_{\Gamma}\right)=\{\text { constant on } \Gamma\}, \quad \operatorname{Im}\left(\bar{\Lambda}_{\Gamma}\right)=\left\{\bar{\lambda} \in W(\Gamma) ; \int_{\Gamma} \bar{\lambda} d H^{1}=0\right\} .
$$


Proof. For $\bar{g}, \bar{h} \in W(\Gamma)$, the solutions of problem (3.2) for boundary values $\bar{g}$ and $\bar{h}$ are denoted by $u \in X_{N}(\bar{g})$ and $w \in X_{N}(\bar{h})$, respectively. Then we obtain

$$
\begin{aligned}
\left(\bar{\Lambda}_{\Gamma}(\bar{g}), \bar{h}\right)_{L^{2}(\Gamma)} & =\sum_{j=1}^{N} \Lambda_{j}(\bar{g}) \int_{\Gamma_{j}} \bar{h} d H^{1}=\sum_{j=1}^{N} \Lambda_{j}(\bar{g}) \int_{\Gamma_{j}} w d H^{1} \\
& =\left(\Lambda_{\Gamma}\left(\gamma_{0} u\right), \gamma_{0} w\right)_{L^{2}(\Gamma)}=a(u, w),
\end{aligned}
$$

and similarly we have $\left(\bar{g}, \bar{\Lambda}_{\Gamma}(\bar{h})\right)_{L^{2}(\Gamma)}=a(u, w)$. These imply the symmetry (3.7). The equality (3.8) follows from (3.5) and (3.6).

It is clear that $\operatorname{Ker}\left(\bar{\Lambda}_{\Gamma}\right) \supset$ \{constant on $\Gamma$. For $\bar{g} \in \operatorname{Ker}\left(\bar{\Lambda}_{\Gamma}\right)$, the solution $u \in X_{N}(\bar{g})$ of (3.2) satisfies $\Lambda_{\Gamma}\left(\gamma_{0} u\right)=0$. From Theorem 2.1, $u$ is constant on $\Gamma$ (and so in $\bar{\Omega}$ ). Thus, it holds that $\operatorname{Ker}\left(\bar{\Lambda}_{\Gamma}\right)=\{$ constant on $\Gamma\}$.

For the image of $\bar{\Lambda}_{\Gamma}$, it satisfies that

$$
\operatorname{Im}\left(\bar{\Lambda}_{\Gamma}\right) \subset \operatorname{Im}\left(\Lambda_{\Gamma}\right) \cap W(\Gamma)=\left\{\bar{\lambda} \in W(\Gamma) ; \int_{\Gamma} \bar{\lambda} d H^{1}=0\right\} .
$$

Since $\operatorname{dim} \operatorname{Im}\left(\bar{\Lambda}_{\Gamma}\right)=\operatorname{dim} W(\Gamma)-\operatorname{dim} \operatorname{Ker}\left(\bar{\Lambda}_{\Gamma}\right)=N-1$, we obtain the latter formula of (3.9).

REMARK 3.4 The extension to the three dimensional case of the above story follows from the parallel argument.

\subsection{General polygonal motion and variational formulas}

In this section, we briefly review a polygonal motion which was proposed in [5]. We consider polygons in $\mathbb{R}^{2}$. Let $P$ be the set of polygonal Jordan curves in $\mathbb{R}^{2}$. In this note, $\Gamma \in P$ is simply called a polygon. For two polygons $\Gamma$ and $\Sigma \in P$, we say $\Gamma \sim \Sigma$, if their numbers of edges are same (let it be $N$ ) and their $j$-th outward unit normal vector $\boldsymbol{n}_{j}$ coincides, $\boldsymbol{n}_{j}(\Gamma)=\boldsymbol{n}_{j}(\Sigma)$ for all $j=1,2, \cdots, N$ after choosing suitable counterclockwise numbering for $\Gamma$ and $\Sigma$.

We fix an $N$-polygon $\Gamma^{*} \in P$ and let $P^{*}:=\left\{\Gamma \in P ; \Gamma \sim \Gamma^{*}\right\}$. For $\Gamma \in P$, we define a height function $\boldsymbol{h}(\Gamma)=\left(h_{1}(\Gamma), h_{2}(\Gamma), \cdots, h_{N}(\Gamma)\right)^{\mathrm{T}} \in \mathbb{R}^{N}$, where $h_{j}(\Gamma)$ is the height from the origin to the $j$-th edge $\Gamma_{j}$ of $\Gamma$.

We consider a polygon $\Gamma(t) \in P^{*}$ parametrized by time $t \in I$, and we call $M:=\bigcup_{t \in I}(\Gamma(t) \times$ $\{t\})$ a polygonal motion in $P^{*}$. This kind of motion has been studied in the context of crystal growth model, where it is called a crystalline motion. See [2, 9, 12, 25-30] and references therein.

For a polygon $\Gamma(t) \in P^{*}$, we denote its interior domain by $\Omega(t)$ with $\partial \Omega(t)=\Gamma(t)$, and denote its height function simply by $\boldsymbol{h}(t)=\left(h_{1}(t), h_{2}(t), \cdots, h_{N}(t)\right)^{\mathrm{T}} \in \mathbb{R}^{N}$. A polygonal motion $M=\bigcup_{t \in I}(\Gamma(t) \times\{t\})$ is called of $C^{k}$-class if $\boldsymbol{h} \in C^{k}\left(I, \mathbb{R}^{N}\right)$. If $M$ is a $C^{1}$-class polygonal motion, the normal velocity $V_{j}$ of $\Gamma_{j}(t)$ is a constant on each $\Gamma_{j}(t)$ and it is given by $V_{j}(t)=\dot{h}_{j}(t)$. We define a normal velocity of $\Gamma(t)$ by $\bar{V}(t):=\sum_{j=1}^{N} V_{j}(t) \chi_{j}(t) \in W(\Gamma(t))$, where $\chi_{j}(t)$ denotes the characteristic function of $\Gamma_{j}(t)$.

Let the $j$-th vertex of $\Gamma \in P^{*}$ be $\boldsymbol{w}_{j}$ and let its outer angle be $\varphi_{j} \in(-\pi, \pi) \backslash\{0\}$, with $\cos \varphi_{j}=\boldsymbol{n}_{j+1} \cdot \boldsymbol{n}_{j}$ as shown in Figure 1. For $\Gamma \in P^{*}$, a polygonal curvature $\kappa_{j}(\Gamma)$ of the $j$-th edge $\Gamma_{j}$ is defined by

$$
\kappa_{j}(\Gamma):=\frac{\eta_{j}}{\left|\Gamma_{j}\right|},
$$


where $\eta_{j}:=\tan \left(\varphi_{j} / 2\right)+\tan \left(\varphi_{j-1} / 2\right)$. For a polygon $\Gamma(t) \in P^{*}$, we define

$$
\bar{\kappa}(t):=\sum_{j=1}^{N} \kappa_{j}(\Gamma(t)) \chi_{j}(t) \in W(\Gamma(t)) .
$$

We have the following polygonal version of variational formulas similar to Theorem 2.2. For a proof of this theorem, see $[4,5]$.

THEOREM 3.5 If $M=\bigcup_{t \in I}(\Gamma(t) \times\{t\})$ is a $C^{1}$-class polygonal motion in $P^{*}$, then $|\Omega(t)|$, $|\Gamma(t)|$ and $c(t)$ belong to $C^{1}(I)$ with respect to $t$ and the following formulas hold for $t \in I$.

1. $\frac{d}{d t}|\Omega(t)|=\sum_{j=1}^{N}\left|\Gamma_{j}(t)\right| V_{j}(t)=\int_{\Gamma(t)} \bar{V} d H^{1}$.

2. $\frac{d}{d t}|\Gamma(t)|=\sum_{j=1}^{N}\left|\Gamma_{j}(t)\right| \kappa_{j}(\Gamma(t)) V_{j}(t)=\int_{\Gamma(t)} \bar{\kappa} \bar{V} d H^{1}$.

3. If $|\Omega(t)|$ is constant in time, $\frac{d}{d t} c(t)=\frac{1}{|\Omega|} \int_{\Gamma(t)} x \bar{V} d H^{1}$.

\subsection{Polygonal Hele-Shaw problem with surface tension}

We consider a polygonal analogue of the Hele-Shaw problem with surface tension (1.1) in this section. Its naive form is given as follows.

Problem 3.6 (Polygonal Hele-Shaw problem) Let $\sigma>0$ and an $N$-polygon $\Gamma^{*} \in P^{*}$ be given. Find a $C^{1}$-class polygonal motion $M=\bigcup_{0 \leqslant t<T}(\Gamma(t) \times\{t\})$ in $P^{*}$ and a pressure filed $p(x, t)$ and a velocity field $\boldsymbol{u}(\boldsymbol{x}, t)$ for $\boldsymbol{x} \in \overline{\Omega(t)}$, such that, for each $t \in[0, T)$,

$$
\begin{cases}V_{j}(t)=\boldsymbol{n}_{j} \cdot \boldsymbol{u}(\boldsymbol{x}, t) & \left(\boldsymbol{x} \in \Gamma_{j}(t), j=1,2, \cdots, N\right), \\ \operatorname{div} \boldsymbol{u}(\boldsymbol{x}, t)=0 & (\boldsymbol{x} \in \Omega(t)), \\ \boldsymbol{u}(\boldsymbol{x}, t)=-\nabla p(\boldsymbol{x}, t) & (\boldsymbol{x} \in \Omega(t)), \\ f_{\Gamma_{j}(t)} p(\boldsymbol{x}, t) d H^{1}=\sigma \kappa_{j}(\Gamma(t)) & (j=1,2, \cdots, N), \\ \Gamma(0)=\Gamma^{*} & \end{cases}
$$

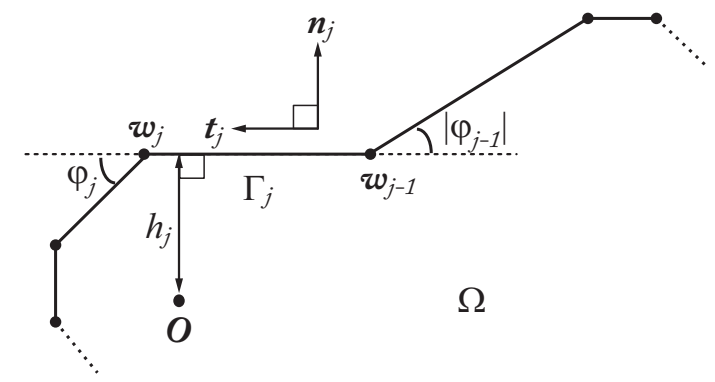

FIG. 1. Outer angles and height around the $j$-th edge $\Gamma_{j}$. The $j$-th height is defined as $h_{j}=\boldsymbol{w}_{j} \cdot \boldsymbol{n}_{j}=\boldsymbol{w}_{j-1} \cdot \boldsymbol{n}_{j}$. 
Using the polygonal Dirichlet-to-Neumann map defined in Section 3.1, we can describe it in the following weak form.

Problem 3.7 (weak form of polygonal HS problem) Let $\sigma>0$ and an $N$-polygon $\Gamma^{*} \in P^{*}$ be given. Find a $C^{1}$-class polygonal motion $M=\bigcup_{0 \leqslant t<T}(\Gamma(t) \times\{t\})$ in $P^{*}$ such that

$$
\left\{\begin{array}{l}
\bar{V}(t)=-\sigma \bar{\Lambda}_{\Gamma(t)}(\bar{\kappa}(t)) \quad(0 \leqslant t<T), \\
\Gamma(0)=\Gamma^{*} .
\end{array}\right.
$$

For a solution of Problem 3.7, for each $t \in[0, T)$, the pressure field $p(t)=p(\cdot, t)$ is constructed as

$$
p(t) \in H^{1}(\Omega(t)) \quad \text { s.t. } \begin{cases}a_{t}(p(t), v)=0 & \left({ }^{\forall} v \in H_{0}^{1}(\Omega(t))\right), \\ \bar{\gamma}_{0} p(t)=\sigma \bar{\kappa}(t) & \text { on } \Gamma(t)\end{cases}
$$

and the velocity field $\boldsymbol{u}(t)=\boldsymbol{u}(\cdot, t)$ is given by (2.5).

This is a polygonal analogue of the original Hele-Shaw problem with a smooth moving boundary.

Theorem 3.8 If $\Gamma(t)(0 \leqslant t<T)$ is a solution of Problem 3.7, the following properties hold.

1. $\frac{d}{d t}|\Omega(t)|=0$

2. $\frac{d}{d t}|\Gamma(t)|=-\frac{1}{\sigma} \int_{\Omega(t)}|\boldsymbol{u}(\boldsymbol{x}, t)|^{2} d \boldsymbol{x} \leqslant 0$

3. $\frac{d}{d t} c(t)=-\sigma|\Omega| \sum_{j=1}^{N} \eta_{j} \boldsymbol{n}_{j}$

Proof. From the formulas of Theorem 3.5, we obtain the area preserving property:

$$
\frac{d}{d t}|\Omega(t)|=\int_{\Gamma(t)} \bar{V} d H^{1}=\langle\bar{V}(t), 1\rangle_{t}=\left\langle-\Lambda_{\Gamma(t)}\left(\gamma_{0} p(t)\right), 1\right\rangle_{t}=-a_{t}(p(t), 1)=0 .
$$

The second property holds as follows.

$$
\begin{aligned}
\frac{d}{d t}|\Gamma(t)| & =\int_{\Gamma(t)} \bar{\kappa} \bar{V} d H^{1}=\sum_{j=1}^{N} V_{j}(t) \int_{\Gamma_{j}(t)} \bar{\kappa} d H^{1} \\
& =\frac{1}{\sigma} \sum_{j=1}^{N} V_{j}(t) \int_{\Gamma_{j}(t)} p d H^{1}=\frac{1}{\sigma} \int_{\Gamma(t)} \bar{V} p d H^{1} \\
& =-\frac{1}{\sigma}\left\langle\Lambda_{\Gamma(t)}\left(\gamma_{0} p(t)\right), \gamma_{0} p(t)\right\rangle_{t}=-\frac{1}{\sigma} a_{t}(p(t), p(t))=-\frac{1}{\sigma} \int_{\Omega(t)}|\boldsymbol{u}(\boldsymbol{x}, t)|^{2} d \boldsymbol{x} .
\end{aligned}
$$

For the center of gravity, from property 1 , we can use formula 3 of Theorem 3.5. For $i=$ $1,2, \cdots, m$, similarly to a proof of Theorem 2.4 , we have

$$
\frac{d}{d t} c_{i}(t)=\int_{\Gamma(t)} \beta_{i} \bar{V} d H^{1}=-\left\langle\Lambda_{\Gamma(t)}\left(\gamma_{0} p(t)\right), \beta_{i}\right\rangle_{t}=-a_{t}\left(p(t), \beta_{i}\right)=-\int_{\Omega(t)} \frac{\partial p}{\partial x_{i}}(\boldsymbol{x}, t) d \boldsymbol{x} .
$$


Hence, we have

$$
\frac{d}{d t} \boldsymbol{c}(t)=-\int_{\Omega(t)} \nabla p(\boldsymbol{x}, t) d \boldsymbol{x}=-\int_{\Gamma(t)} p \boldsymbol{n} d H^{1}=-\sum_{j=1}^{N} \sigma \kappa_{j}(\Gamma(t))\left|\Gamma_{j}(t)\right| \boldsymbol{n}_{j}=-\sigma \sum_{j=1}^{N} \eta_{j} \boldsymbol{n}_{j} .
$$

REMARK 3.9 We remark that if we choose our polygon class $P^{*}$ with the condition $\sum_{j=1}^{N} \eta_{j} \boldsymbol{n}_{j}=$ 0, then

$$
\frac{d}{d t} c(t)=\mathbf{0}
$$

holds.

\section{Local solvability of polygonal Hele-Shaw problem}

In this section, we prove time local solvability and uniqueness of the solution of polygonal HeleShaw problem (Problem 3.7). We first prepare some necessary notation and lemmas.

For a fixed $\Gamma^{*} \in P$, we define an open set:

$$
O^{*}:=\left\{\boldsymbol{h}(\Gamma) ; \Gamma \sim \Gamma^{*}\right\} \subset \mathbb{R}^{N} .
$$

Then $P^{*}$ and $O^{*}$ correspond to each other by the mapping $P^{*} \ni \Gamma \mapsto \boldsymbol{h}(\Gamma) \in O^{*}$. The inverse mapping from $O^{*}$ to $P^{*}$ is denoted by

$$
\Gamma(\boldsymbol{h}):=\Gamma \quad\left(\boldsymbol{h} \in O^{*}, \boldsymbol{h}=\boldsymbol{h}(\Gamma), \Gamma \in P^{*}\right) .
$$

The $j$-th vertex of $\Gamma(\boldsymbol{h})$ is denoted by $\boldsymbol{w}_{j}(\boldsymbol{h}) \in \mathbb{R}^{2}$, and the interior domain of $\Gamma(\boldsymbol{h})$ is denoted by $\Omega(\boldsymbol{h})$. For $\boldsymbol{h} \in O^{*}$, we also denote the polygonal curvature of $\Gamma=\Gamma(\boldsymbol{h}) \in P^{*}$ by

$$
\bar{\kappa}(\boldsymbol{h})=\sum_{j=1}^{N} \kappa_{j}(\boldsymbol{h}) \chi_{j} \in W(\Gamma) .
$$

It is clear that $\kappa_{j} \in C^{\infty}\left(O^{*}\right)(j=1,2, \cdots, N)$.

Similarly to Proposition 2.1 of [4], we can construct a family of Lipschitz mappings $\Phi(\boldsymbol{h})=$ $\Phi(\cdot, \boldsymbol{h})$ from a fixed domain to $\Omega(\boldsymbol{h})$. We take $\hat{\boldsymbol{h}} \in O^{*}$ arbitrarily and fix it. We define $\hat{\Gamma}:=\Gamma(\hat{\boldsymbol{h}})$ and denote the interior polygonal domain of $\hat{\Gamma}$ by $\hat{\Omega}$. The closure of $\hat{\Omega}$ is denoted by $\hat{\Omega^{\wedge}}$

LEMMA 4.1 For $\hat{\boldsymbol{h}} \in O^{*}$ and a bounded domain $Q \supset \overline{\Omega^{*}}$, there exist an open set $O \subset O^{*}$ with $\hat{\boldsymbol{h}} \in O$ and a family of domain mapping $\Phi \in C^{\infty}\left(O, W^{1, \infty}\left(Q, \mathbb{R}^{2}\right)\right)$, and they satisfy the following conditions.

1. $\Phi(\boldsymbol{h})$ is a bi-Lipschitz transform from $\bar{Q}$ onto itself, i.e., $\Phi(\boldsymbol{h})$ is bijective from $\bar{Q}$ onto itself and $\Phi(\boldsymbol{h})$ and $\Phi(\boldsymbol{h})^{-1}$ are both Lipschitz continuous on $\bar{Q}$.

2. $\Phi(\boldsymbol{x}, \boldsymbol{h})=\boldsymbol{x}$ for $\boldsymbol{x}$ in a neighborhood of $\partial Q$ for $\boldsymbol{h} \in O$.

3. $\Phi\left(\overline{\Omega^{\wedge}}, \boldsymbol{h}\right)=\overline{\Omega(\boldsymbol{h})}$ for $\boldsymbol{h} \in O$, and $\Phi(\boldsymbol{h})$ is an affine map from each edge $\hat{\Gamma}_{j}$ onto $\Gamma_{j}(\boldsymbol{h})$ with $\Phi\left(\boldsymbol{w}_{j}(\hat{\boldsymbol{h}}), \boldsymbol{h}\right)=\boldsymbol{w}_{j}(\boldsymbol{h})$, i.e.,

$$
\Phi\left((1-\theta) \boldsymbol{w}_{j-1}(\hat{\boldsymbol{h}})+\theta \boldsymbol{w}_{j}(\hat{\boldsymbol{h}}), \boldsymbol{h}\right)=(1-\theta) \boldsymbol{w}_{j-1}(\boldsymbol{h})+\theta \boldsymbol{w}_{j}(\boldsymbol{h}) \quad(j=1,2, \cdots, N, \theta \in[0,1]) .
$$


Proof. Without loss of generality, we assume that $Q$ is a bounded polygonal domain. We consider a triangulation $T$ of $\hat{\Omega}$ and $Q$ which satisfies the following conditions.

(1) $T=\{K\}$ is a finite collection of closed triangles $K \subset \mathbb{R}^{2}$, and $\bar{Q}=\bigcup_{K \in T} K$.

(2) If $K, K^{\prime} \in T$ and $K \neq K^{\prime}, K \cap K^{\prime}$ is any one of the empty set, a common vertex or a common edge of $K$ and $K^{\prime}$.

(3) There exists a subset $T_{0} \subset T$ such that $\overline{\Omega^{\wedge}}=\bigcup_{K \in T_{0}} K$, and $N \cap \hat{\Gamma}=\left\{\boldsymbol{w}_{j}(\hat{\boldsymbol{h}})\right\}_{j=1}^{N}$, where $N$ denotes the set of all vertices of triangles in $T$.

(4) There does not exist any $K \in T$ with $K \cap \hat{\Gamma} \neq \emptyset$ and $K \cap \partial Q \neq \emptyset$.

We assume that $\Phi(\boldsymbol{h})=\Phi(\cdot, \boldsymbol{h}) \in C^{0}\left(\bar{Q}, \mathbb{R}^{2}\right)$ and that it has the following form:

$$
\Phi(\boldsymbol{x}, \boldsymbol{h})=A_{K}(\boldsymbol{h})\left(\begin{array}{c}
\boldsymbol{x} \\
1
\end{array}\right) \quad(\boldsymbol{x} \in K \in T),
$$

where $A_{K}(\boldsymbol{h})$ is a $2 \times 3$ matrix depending on $K \in T$ and $\boldsymbol{h} \in O$. To determine $A_{K}(\boldsymbol{h})$, we suppose the condition:

$$
\Phi(\boldsymbol{x}, \boldsymbol{h})= \begin{cases}\boldsymbol{x} & \text { if } \boldsymbol{x} \in N \backslash \hat{\Gamma}, \\ \boldsymbol{w}_{j}(\boldsymbol{h}) & \text { if } \boldsymbol{x}=\boldsymbol{w}_{j}(\hat{\boldsymbol{h}}) \in N \cap \hat{\Gamma} .\end{cases}
$$

For sufficiently small $O, \Phi(\boldsymbol{x}, \boldsymbol{h})$ is uniquely determined by the conditions (4.1) and (4.2) for $\boldsymbol{h} \in$ $O$. It is also clear that $\Phi(\boldsymbol{h}) \in W^{1, \infty}\left(Q, \mathbb{R}^{2}\right)$ is bijective from $Q$ onto itself and $\Phi(\boldsymbol{x}, \boldsymbol{h})=\boldsymbol{x}$ for $x \in K$ if $K \cap \hat{\Gamma}=\emptyset$.

Let us fix $K \in T$ and let $\boldsymbol{x}_{1}, \boldsymbol{x}_{2}, \boldsymbol{x}_{3}$ be the three vertices of $K$. Then, from (4.2), $\boldsymbol{y}_{l}(\boldsymbol{h}):=$ $\Phi\left(\boldsymbol{x}_{l}, \boldsymbol{h}\right)$ satisfies the condition $\boldsymbol{y}_{l} \in C^{\infty}\left(O, \mathbb{R}^{2}\right)$ for $l=1,2,3$. Since

$$
\boldsymbol{y}_{l}(\boldsymbol{h})=A_{K}(\boldsymbol{h})\left(\begin{array}{c}
\boldsymbol{x}_{l} \\
1
\end{array}\right) \quad(l=1,2,3),
$$

we have

$$
A_{K}(\boldsymbol{h})=\left(y_{1}(h), y_{2}(h), y_{3}(h)\right)\left(\begin{array}{ccc}
x_{1} & x_{2} & x_{3} \\
1 & 1 & 1
\end{array}\right)^{-1} .
$$

From this expression, we obtain $A_{K} \in C^{\infty}\left(O, \mathbb{R}^{2 \times 3}\right)$. By the above construction of $\Phi$, the three conditions of the lemma are all satisfied. The regularity condition $\Phi \in C^{\infty}\left(O, W^{1, \infty}\left(Q, \mathbb{R}^{2}\right)\right)$ follows from (4.1) and $A_{K} \in C^{\infty}\left(O, \mathbb{R}^{2 \times 3}\right)$.

LEMMA 4.2 We define

$$
\lambda_{i j}(\boldsymbol{h}):=\Lambda_{i}\left(\chi_{j}\right) \quad \text { on } \Gamma=\Gamma(\boldsymbol{h}) \quad\left(\boldsymbol{h} \in O^{*}, i, j=1,2, \cdots, N\right) .
$$

Then $\lambda_{i j} \in C^{\infty}\left(O^{*}\right)$ holds for $i, j=1,2, \cdots, N$.

Proof. We fix $\hat{\boldsymbol{h}} \in O^{*}$ and take $O \subset O^{*}$ and $\Phi \in C^{\infty}\left(O, W^{1, \infty}\left(Q, \mathbb{R}^{2}\right)\right)$ as given in Lemma 4.1. For $i, j=1,2, \cdots, N$, we show that $\lambda_{i j} \in C^{\infty}(O)$ below. To apply the theory of the polygonal Dirichlet-to-Neumann map in Section 3.1, we denote $a(u, v)$ and $X_{N}(\bar{g})$ on $\Omega$ by $a_{\Omega}(u, v)(u, v \in$ $\left.H^{1}(\Omega)\right)$ and $X_{N}(\bar{g}, \Omega)(\bar{g} \in W(\partial \Omega))$, respectively. We, however, denote the characteristic function of $\Gamma_{j}(\boldsymbol{h})$ by $\chi_{j} \in W(\Gamma(\boldsymbol{h}))$ for simplicity. 
We define the push-forward operator from $\hat{\Omega}$ onto $\Omega(\boldsymbol{h})$ :

$$
\Phi(\boldsymbol{h})_{*}: \hat{v} \longmapsto v=\hat{v} \circ \Phi(\boldsymbol{h})^{-1} \quad\left(\hat{v}=v \circ \Phi(\boldsymbol{h}) \in H^{1}(\hat{\Omega})\right) .
$$

We denote the space variables on $\Omega(\boldsymbol{h})$ and $\hat{\Omega}$ by $\boldsymbol{x}$ and $\hat{\boldsymbol{x}}$, respectively. Then, for $\boldsymbol{h} \in O$, we have

$$
\begin{array}{ll}
\boldsymbol{x}=\Phi(\hat{\boldsymbol{x}}, \boldsymbol{h}) \in \Omega(\boldsymbol{h}) & (\hat{\boldsymbol{x}} \in \hat{\Omega}) \\
\Phi(\boldsymbol{h})_{*} \hat{v}(\boldsymbol{x})=\hat{v}(\hat{\boldsymbol{x}}) & \left(\hat{v} \in H^{1}(\hat{\Omega}), \hat{\boldsymbol{x}} \in \hat{\Omega}\right) .
\end{array}
$$

We obtain the formula:

$$
\nabla\left(\Phi(\boldsymbol{h})_{*} \hat{v}\right)(\boldsymbol{x})=A(\hat{\boldsymbol{x}}, \boldsymbol{h}) \nabla \hat{v}(\hat{\boldsymbol{x}}) \quad\left(\hat{v} \in H^{1}(\hat{\Omega}), \hat{\boldsymbol{x}} \in \hat{\Omega}\right),
$$

where

$$
A(\hat{\boldsymbol{x}}, \boldsymbol{h}):=\left(\nabla \Phi(\hat{\boldsymbol{x}}, \boldsymbol{h})^{\mathrm{T}}\right)^{-1} \in \mathbb{R}^{2 \times 2} \quad(\hat{\boldsymbol{x}} \in \hat{\Omega}, \boldsymbol{h} \in O) .
$$

Since $\Phi(\boldsymbol{h})$ is a bi-Lipschitz domain mapping from $\hat{\Omega}$ onto $\Omega(\boldsymbol{h})$, the push-forward operator $\Phi(\boldsymbol{h})_{*}$ becomes a linear topological isomorphism from $H^{1}(\hat{\Omega})$ onto $H^{1}(\Omega(\boldsymbol{h}))$. See Section 3 in [19] or Section 7 in [17] for further details.

In particular, since $\Phi(\boldsymbol{h})$ is an affine mapping on each $\hat{\Gamma}_{j}$,

$$
f_{\hat{\Gamma}_{j}} \hat{v} d H^{1}=f_{\Gamma_{j}(\boldsymbol{h})} \Phi(\boldsymbol{h})_{*} \hat{v} d H^{1} \quad\left(\hat{v} \in H^{1}(\hat{\Omega}), j=1,2, \cdots, N\right)
$$

hold and $\Phi(\boldsymbol{h})_{*}$ becomes a linear topological isomorphism from $X_{N}\left(\chi_{i}, \hat{\Omega}\right)$ onto $X_{N}\left(\chi_{i}, \Omega(\boldsymbol{h})\right)$.

We define a pull-back of the bilinear form:

$$
\hat{a}(\hat{u}, \hat{v}, \boldsymbol{h}):=a_{\Omega(\boldsymbol{h})}\left(\Phi(\boldsymbol{h})_{*} \hat{u}, \Phi(\boldsymbol{h})_{*} \hat{v}\right) \quad\left(\hat{u}, \hat{v} \in H^{1}(\hat{\Omega}), \boldsymbol{h} \in O\right) .
$$

Then we have

$$
\begin{aligned}
\hat{a}(\hat{u}, \hat{v}, \boldsymbol{h}) & =\int_{\Omega(\boldsymbol{h})} \nabla\left(\Phi(\boldsymbol{h})_{*} \hat{u}\right) \cdot \nabla\left(\Phi(\boldsymbol{h})_{*} \hat{v}\right) d \boldsymbol{x} \\
& =\int_{\hat{\Omega}}(A(\hat{\boldsymbol{x}}, \boldsymbol{h}) \nabla \hat{u}(\hat{\boldsymbol{x}})) \cdot(A(\hat{\boldsymbol{x}}, \boldsymbol{h}) \nabla \hat{v}(\hat{\boldsymbol{x}})) \hat{J}(\hat{\boldsymbol{x}}, \boldsymbol{h}) d \hat{\boldsymbol{x}},
\end{aligned}
$$

where $\hat{J}$ is the Jacobian defined as

$$
\hat{J}(\hat{\boldsymbol{x}}, \boldsymbol{h}):=\operatorname{det} \nabla \Phi(\hat{\boldsymbol{x}}, \boldsymbol{h})^{\mathrm{T}} \quad(\hat{\boldsymbol{x}} \in \hat{\Omega}, \boldsymbol{h} \in O) .
$$

We remark that $A \in C^{\infty}\left(O, L^{\infty}\left(\hat{\Omega}, \mathbb{R}^{2 \times 2}\right)\right)$ and $\hat{J} \in C^{\infty}\left(O, L^{\infty}(\hat{\Omega})\right)$ hold (see [17, 19], again).

From Theorem 3.3, we obtain

$$
\lambda_{i j}(\boldsymbol{h})=\frac{a_{\Omega(\boldsymbol{h})}\left(u_{j}(\boldsymbol{h}), v_{i}\right)}{\left|\Gamma_{j}(\boldsymbol{h})\right|} \quad(\boldsymbol{h} \in O),
$$

where

$$
u_{j}(\boldsymbol{h}):=\underset{v \in X_{N}\left(\chi_{j}, \Omega(\boldsymbol{h})\right)}{\arg \min } a_{\Omega(\boldsymbol{h})}(v, v) \quad(\boldsymbol{h} \in O),
$$


and $v_{i}$ is an arbitrary function satisfying

$$
v_{i} \in X_{N}\left(\chi_{i}, \Omega(\boldsymbol{h})\right) .
$$

We denote the pull-back of $v_{i}$ from $\Omega(\boldsymbol{h})$ to $\hat{\Omega}$ by $\hat{v}_{i}=v_{i} \circ \Phi(\boldsymbol{h})$. Then (4.5) is equivalent to $\hat{v}_{i} \in X_{N}\left(\chi_{i}, \hat{\Omega}\right)$. We also define $\hat{u}_{j}(\boldsymbol{h}):=u_{j}(\boldsymbol{h}) \circ \Phi(\boldsymbol{h})$. Then we have

$$
\hat{u}_{j}(\boldsymbol{h})=\underset{\hat{v} \in X_{N}\left(\chi_{j}, \hat{\Omega}\right)}{\arg \min } \hat{a}(\hat{v}, \hat{v}, \boldsymbol{h}),
$$

and

$$
\lambda_{i j}(\boldsymbol{h})=\frac{\hat{a}\left(\hat{u}_{j}(\boldsymbol{h}), \hat{v}_{i}, \boldsymbol{h}\right)}{\left|\Gamma_{j}(\boldsymbol{h})\right|} \quad(\boldsymbol{h} \in O) .
$$

From the expression (4.4), it follows that the mapping $[(\hat{v}, \boldsymbol{h}) \longmapsto \hat{a}(\hat{v}, \hat{v}, \boldsymbol{h})]$ belongs to $C^{\infty}\left(H^{1}(\hat{\Omega}) \times O, \mathbb{R}\right)$. Thus we can apply Theorem 2.5 of [19] to the minimization problem (4.6) and obtain that $\hat{u}_{j} \in C^{\infty}\left(O, H^{1}(\hat{\Omega})\right)$.

We fix $\hat{v}_{i} \in X_{N}\left(\chi_{i}, \hat{\Omega}\right)$. The assertion $\lambda_{i j} \in C^{\infty}(O)$ follows from the formula (4.7) and the regularities:

$$
\begin{aligned}
{\left[(\hat{v}, \boldsymbol{h}) \longmapsto \hat{a}\left(\hat{v}, \hat{v}_{i}, \boldsymbol{h}\right)\right] } & \in C^{\infty}\left(H^{1}(\hat{\Omega}) \times O, \mathbb{R}\right), \\
{\left[\boldsymbol{h} \longmapsto \hat{u}_{j}(\boldsymbol{h})\right] } & \in C^{\infty}\left(O, H^{1}(\hat{\Omega})\right), \\
{\left[\boldsymbol{h} \longmapsto\left|\Gamma_{j}(\boldsymbol{h})\right|\right] } & \in C^{\infty}(O, \mathbb{R}) .
\end{aligned}
$$

Using this lemma, we can show local existence of a solution of the polygonal Hele-Shaw problem.

Theorem 4.3 For Problem 3.7, there exists a local solution $M=\bigcup_{0 \leqslant t<T}(\Gamma(t) \times\{t\})$ of $C^{1}$-class polygonal motion in $P^{*}$ with some $T \in(0, \infty]$. Moreover the solution is unique and it becomes a polygonal motion of $C^{\infty}$-class.

Proof. Problem 3.7 is equivalent to the following initial value problem of a system of ODEs:

$$
\left\{\begin{aligned}
\frac{d h_{i}}{d t} & =-\sigma \sum_{j=1}^{N} \lambda_{i j}(\boldsymbol{h}) \kappa_{j}(\boldsymbol{h}) \quad(i=1,2, \cdots, N) \\
\boldsymbol{h}(0) & =\boldsymbol{h}\left(\Gamma^{*}\right),
\end{aligned}\right.
$$

where $\lambda_{i j}(\boldsymbol{h})$ is defined by (4.3). Since $\lambda_{i j}$ and $\kappa_{j}$ belong to $C^{\infty}\left(O^{*}\right)$, the assertion of the theorem follows from the standard theory of ordinary differential equations.

\section{Conclusions}

In this paper, we first revealed some precise variational structures of the Hele-Shaw moving boundary problem with surface tension (1.1) in Section 2, namely, the Dirichlet-to-Neumann map 
formulation in Problem 2.3 and some variational properties in Theorem 2.4. In the theorem, we have shown properties of area preservation, curve shortening, and preservation of the center of gravity.

In Section 3, based on the idea of [5], we proposed a polygonal analogue of the Hele-Shaw problem in Problem 3.6, and we have shown that its weak formulation is given by introducing the polygonal Dirichlet-to-Neumann map. Furthermore, we have shown in Theorem 3.8 that the polygonal Hele-Shaw problem admits the same variational structures as ones in Theorem 2.4. In the last section, we proved time local existence of a unique solution of the polygonal Hele-Shaw problem.

These results of this paper together with [5] suggest that the method of crystalline motion or polygonal motion can provide an effective discrete model not only for the classical crystal growth but also for various moving boundary problems with variational structures. Our polygonal motion approach enables us to handle the variational structures of the original problem including the curvature term in an easier semi-discrete system.

Acknowledgements. The authors would like to thank the anonymous referees for their comments and suggestions. The second and the third authors were supported by Grant-in-Aid for Young Scientists (B) 21740078 (DT) and Grant-in-Aid for Scientific Research (C) 23540150 (SY).

\section{REFERENCES}

1. Aitchison, J. M. And Howison, S. D., Computation of Hele-Shaw flows with free boundaries. $J$. Comput. Phys. 60 (1985), 376-390. Zb10573.76043 Mr0814435

2. Almgren, F. And TAYlor, J. E., Flat flow is motion by crystalline curvature for curves with crystalline energies. J. Diff. Geom. 42 (1995), 1-22. Zb10867.58020 MR1350693

3. Almgren, R., Crystalline Saffman-Taylor fingers. SIAM J. Appl. Math. 55 (1995), 1511-1535. Zb10838.76094 MR1358787

4. Beneš, M., Kimura, M. And Yazaki, S., Analytical and numerical aspects on motion of polygonal curves with constant area speed. MAGIA 2007 Proceedings, Slovak University of Technology in Bratislava (2007), 127-141.

5. Beneš, M., Kimura, M. And YAZAKi, S., Second order numerical scheme for motion of polygonal curves with constant area speed. Interfaces and Free Boundaries 11 (2009), 515-536. Zbl1189.65189 MR2576215

6. Chen, X., Hong, J. And Yi, F., Existence, uniqueness, and regularity of classical solutions of the Mullins-Sekerka problem. Comm. Partial Diff. Eq. 21 (1996), 1705-1727. MR1421209

7. Duchon, J. And Robert, R., Évolution d'une interface par capillarité et diffusion de volume I. Existence locale en temps. Ann. Inst. Henri Poincaré, Analyse non linéaire 1 (1984), 361-378. Zb10572. 35051 MR0779874

8. Escher, J. And Simonett, G., Classical solutions for Hele-Shaw models with surface tension. Advances in Differential Equations 2 (1997), 619-642. Zbl1023.35527 MR1441859

9. Giga, M.-H. AND GigA, Y., Crystalline and level set flow-convergence of a crystalline algorithm for a general anisotropic curvature flow in the plane. Free boundary problems: theory and applications, I (Chiba, 1999), Gakkotosho (2000), 64-79. Zbl0957.35122 MR1793023

10. Giga, Y. AND RYBKA, P., Quasi-static evolution of 3-D crystals grown from supersaturated vapor. Differential Integral Equations 15 (2002), 1-15. Zbl1038.35176 MR1869818

11. Giga, Y. And RyBKA, P., Stability of facets of crystals growing from vapor. Discrete Contin. Dyn. Syst. 14 (2006), 689-706. Zbl1095. 35078 MR2177092 
12. GIRÃO, P. M., Convergence of a crystalline algorithm for the motion of a simple closed convex curve by weighted curvature. SIAM J. Numer. Anal. 32 (1995), 886-899. Zb10830.65150 MR1335660

13. Girault, V. And Raviart, P.-A., Finite Element Methods for Navier-Stokes Equations, Theory and Algorithms: Springer Series in Computational Mathematics, Vol. 5. Springer (1986). Zb10585.65077 MR0851383

14. Hele-Shaw, H. S., The flow of water. Nature58 (1897), 467-468. JFM 29.0646.01

15. Kimura, M., Numerical analysis for moving boundary problems using the boundary tracking method. Japan J. Indust. Appl. Math. 14 (1997), 373-398. Zbl0892.76065 MR1475140

16. Kimura, M., Time local existence of a moving boundary of the Hele-Shaw flow with suction. Euro. J. Appl. Math. 10 (2000), 581-605. Zbl0955.76020 MR1757943

17. Kimura, M., Shape derivative of minimum potential energy: Abstract theory and applications. Jindřich Nečas Center for Mathematical Modeling Lecture notes Volume IV, Topics in Mathematical Modeling (2008), 1-38. MR2868563

18. Kimura, M., Geometry of hypersurfaces and moving hypersurfaces in $\mathbf{R}^{m}-$ for the study of moving boundary problems -. Jindřich Nečas Center for Mathematical Modeling Lecture notes Volume IV, Topics in Mathematical Modeling (2008), 39-93. MR2868564

19. Kimura, M. AND WAKANO, I., Shape derivative of potential energy and energy release rate in fracture mechanics. Journal of Math-for-industry 3 (2011) A, 21-31. Zbl1256. 49055 MR2788704

20. LAMB, H., Hydrodynamics. 6th ed., Cambridge Univ. Press (1932). JFM 58.1298.04

21. PAterson, L., Radial fingering in a Hele-Shaw cell. J. Fluid Mech. 113 (1981), 513-529.

22. RiCHARDSON, S., Hele-Shaw flows with a free boundary produced by the injection of fluid into a narrow channel. J. Fluid Mech. 56 (1972), 609-618. Zbl0256.76024

23. Shelley, M. J., Tian, F.-R. And Wlodarski, K., Hele-Shaw flow and pattern formation in a timedependent gap. Nonlinearity 10 (1997), 1471-1495. Zb10911.76026 MR1483552

24. Tabeling, P. , Zocchi, G. And Libchaber, A., An experimental study of the Saffman Taylor instability. J. Fluid Mech. 177 (1987), 67-82.

25. TAYlor, J. E., Constructions and conjectures in crystalline nondifferential geometry. Proceedings of the Conference on Differential Geometry, Rio de Janeiro, Pitman Monographs Surveys Pure Appl. Math. 52 (1991), 321-336. Zbl0725.53011 MR1173051

26. TAYLOR, J. E., Motion of curves by crystalline curvature, including triple junctions and boundary points. Differential geometry: Partial differential equations on manifolds (Los Angeles, CA, 1990), Proc. Sympos. Pure Math. 54, Part 1, Amer. Math. Soc. (1993), 417-438. Zb10823. 49028 MR1216599

27. Taylor, J. E., Cahn, J. And Handwerker, C., Geometric models of crystal growth. Acta Metall. 40 (1992), 1443-1474.

28. UshiJima, T. K. AND YAZAKI, S., Convergence of a crystalline approximation for an area-preserving motion. J. Comput. Appl. Math. 166 (2004), 427-452. Zbl1052.65082 MR2041191

29. YAZAKI, S., On an area-preserving crystalline motion. Calc. Var. 14 (2002), 85-105. Zbl1143. 37320 MR1883601

30. YAZAKI, S., Motion of nonadmissible convex polygons by crystalline curvature. Publications of Research Institute for Mathematical Sciences 43 (2007), 155-170. Zbl1132.53036 MR2317117 